\title{
How to Utilize L. J.S. Allen's "An Introduction to Mathematical Biology" in a Biomathematics Course
}

\author{
Füsun Akman ${ }^{1, *}$
}

\begin{abstract}
This is a combined textbook review and course plan for a biomathematics modeling course that is taught at the author's home institution as a foundation course in the Biomathematics Master's Program. The pros and cons of using Linda J. S. Allen's textbook, An Introduction to Mathematical Biology, for a one-semester course is discussed. Moreover, strategies for coping with some problems that may arise are proposed.
\end{abstract}

Keywords: biomathematics course, textbook choice, modeling with difference equations, modeling with differential equations

\section{Introduction}

I have taught "Quantitative Mathematics" (MAT 442) several times as a three-credit-hour foundation course for the Biomathematics Master's Program at my home institution. Both Allen's book, An Introduction to Mathematical Biology [1], and Edelstein-Keshet's book, Mathematical Models in Biology [4], were used as the main textbook in different semesters. It is important to point out that these textbooks, written by eminent authorities in the field, are still the only ones that satisfy most of the requirements of MAT 442 among the many that have been published before and since. The catalog description of the course includes an introduction to difference equations as well as biomathematical modeling with difference, ordinary differential, and partial differential equations, assuming familiarity with differential equations and linear algebra. However, the constraints of introducing difference equations from scratch, including as many meaningful examples as possible, and satisfying the needs of a non-homogeneous audience - consisting of graduate students in mathematics and biology as well as advanced undergraduates - in one semester are overwhelming, and neither textbook is completely adequate in its treatment of all course objectives.

I will briefly describe the contents of Allen's book [1], point out the pros and cons of using it as a textbook for a course similar to ours, and suggest ways of augmenting the presentation for the benefit of the students. In addition, I will propose a course outline for first-time users that involves mainly population biology, a compromise that was necessary for me to be able to put together a unified and complete course. In my opinion, the extra work is justified when compared to the alternative of patching many different resources or using a textbook that lacks the solid introduction as well as the variety provided by Allen (or Edelstein-Keshet). Ideally, difference equations or nonlinear systems should be taught in a separate course, but it is possible to do without. I contend that whether used for an established course or adopted for an experimental elective, this textbook should serve its

\footnotetext{
${ }^{1}$ Department of Mathematics, Illinois State University, Normal, IL 61790-4520

${ }^{*}$ Correspondence: akmanf $@ i l s t u . e d u$
} 
purpose well, with the caveats expanded upon in this article.

\section{The Subject Matter}

\subsection{Table of Contents}

The table of contents of Allen [1] was, in fact, used as an inspiration for the catalog description of MAT 442:

1. Linear difference equations, theory, and examples

2. Nonlinear difference equations, theory, and examples

3. Biological applications of difference equations

4. Linear differential equations: theory and examples

5. Nonlinear ordinary differential equations: theory and examples

6. Biological applications of differential equations

7. Partial differential equations: theory, examples, and applications

The book, according to the Preface, has been used for a one-semester graduate course at Texas Tech University. It covers deterministic models only (for stochastic models, we refer the reader to Allen [2]), and the "[m]athematical prerequisites include undergraduate courses in calculus, linear algebra, and differential equations." As we shall see, this is a strict inclusion in the set theory sense, and some background in physics, chemistry, and biology is also necessary for an ambitious instructor whose training is mainly in mathematics. "The emphasis is on predicting the qualitative solution behavior over time."

\section{$2.2 \quad$ Pros}

\subsubsection{General}

Allen [1] is one of the rare books I have found that treats difference equations as a complete subject while being able to gain depth quickly in the subject of modeling with such equations. ODE's and PDE's are treated later, assuming some acquaintance, but still reviewing the solutions of the basic classes of equations that are necessary to follow the book. It is an incredible feat to have compressed the theory and a large number of examples, exercises, and a separate bibliography for each chapter, as well as techniques of solutions, into a thin volume. The typesetting is reader-friendly, the graphics are clear, and there are no annoying gimmicks such as unnecessary pictures and irrelevant boxes of information. A Solutions Manual is also available. It is possible to support the course with any technology preferred by the instructor, from Microsoft Excel to MATLAB and Maple, for which code is provided within the main text and in the appendices.

It is clearly impossible to cover every contemporary modeling technique in an introductory textbook, but the ones chosen by the author are classical, varied, and balanced. Allen does refer to the fact that many examples are from population biology and epidemiology due to her own field of expertise; however, this is an advantage for an introductory course where students would have a common experience in recognizing the subject matter and having been exposed to the ideas in real life. For the courageous instructor, there is always the opportunity to discuss Michaelis-Menten kinetics or excitable systems. More sections can be covered in a four-credit-hour or two-semester course, and the treatment can be as advanced as desired with students of a stronger background. On the other hand, the textbook can be adapted for a self-contained honors undergraduate course, with a careful selection of topics. It is also a well-used reference book for researchers in biomathematics. Allen has clearly done her field a valuable service by creating this near-encyclopedia of theory and applications, which is much greater than the sum of its parts. 


\subsubsection{Technical}

I especially appreciate the fact that the concept of stability is handled properly. Many other textbooks at undergraduate or graduate level do not distinguish between locally stable (LS) and locally attracting (LA) equilibria; rather, they bundle the two conditions under one name, "stable", where they may mean locally attracting, locally stable, or most possibly locally asymptotically stable (LAS), the latter representing the desirable case of being LA and LS at the same time. Many a time an author blurs the distinction for pedagogical purposes, but ends up with an example where additional explanations need to be made, confusing the reader. The difference may a life-or-death issue, as illustrated in the wellknown example: suppose that the Earth gets thrown slightly off-orbit by a nearby comet's gravitational pull. If its orbit were merely locally stable to begin with, then it would stay close to the original for the following years, but the deviation may still be too much for life to survive, or it will at the very least cause mass extinctions and severe adaptation problems for humankind. If the orbit were only locally attracting but not locally stable, then the good news is that we would eventually be settled in our current orbit. The bad news is that straying far away even once may wipe out all life! More mundane applications of the same principles affect satellites orbiting the Earth, as well as the fates of specific populations of wildlife, under small perturbations. Allen not only emphasizes the distinction but provides simple examples where one condition holds to the exclusion of the other as well (see Examples 2.2 and 2.7).

The proofs of certain hard theorems (but not all; see "Cons") in real analysis are confined to the appendices of the chapters, which is a necessity as more and more students matriculate with AP Calculus credentials and may not have been exposed to epsilon-delta proofs in a rigorous analysis course. One such example is Theorem 2.6, tying the existence of a globally asymptotically stable equilibrium to the non-existence of 2-cycles. A compromise approach is used (for example, for Theorem 4.4, the Routh-Hurwitz Criteria) when the general proof is lengthy, but the simplest case can be verified in a few short paragraphs.

The exceptional cases of certain theorems -again, not all- are studied meticulously, such as the "nonhyperbolic" equilibria for which the standard test for LAS (involving the derivative of the recursive function; Theorem 2.1) does not apply. Tucked at the end of the section on local stability in first-order difference equations, the treatment of the Schwartzian derivative allows an instructor to include or exclude the nonhyperbolic case, or an interested student to find further information if not covered in class.

\subsection{Cons}

Some prior planning and re-organization is essential: unless one is a veteran practitioner of the art, the book is not easy to teach from directly. A mixed audience makes it necessary to cover some basic organizing principles and concepts from mathematics, biology, and biochemistry.

\subsubsection{Organization and Unity}

Although the textbook is fairly uncluttered and pleasing to the eye, closer inspection reveals that the relative importance and length, as well as the flavor (as in "definition", "theorem", "observation", "example", "fact from biology", or "short free-floating computation"), of units of information could be organized more effectively. An example may be mentioned briefly in a paragraph, be isolated as a numbered example, or be spread over several sections; e.g., Leslie's age-structured model takes up Sections 1.6 and 1.7. Some definitions are boxed in, whereas some are mentioned in passing, such as that of "bifurcation diagram" on page 57 or "basic reproduction number" on page 71. Sections and subsections seem to be arbitrary dividing points rather than tools of organizing chunks of information that are roughly on the same footing. Chaotic nonlinear systems and various related subjects are mixed up with applications instead of initially being given their own space. My remedy was to write 
complete lecture notes based on the textbook and post them for the students to download. Obviously, any set of handouts hitting some point of the scale between doing nothing and my solution (with proper divisions and headings) can be used and is highly recommended, both for advance course planning and for informing the students about what is to be covered.

\subsubsection{Missing or misleading information}

Some examples, keeping in mind the goals for courses similar to our MAT 442, are as follows.

1. In solving first-order homogeneous linear difference equations of the form $X_{t+1}=A X_{t}$, where $A$ is a constant coefficient matrix, the cases of complex eigenvalues, non-diagonalizable matrices, and matrices of size larger than two-by-two are not treated completely (see page 15). This also affects the solutions of nonlinear first-order systems via their Jacobians. The instructor may want to provide a more detailed handout of the theory with examples of these exceptional cases.

2. The solution of the system $X_{t+1}=A X_{t}$ of difference equations in Section 1.5 is only given in terms of the eigenvalues and eigenvectors for a diagonalizable matrix $A$. The alternative solution $X_{t}=A^{t} X_{0}$ is more elegant and should probably be mentioned first as the natural extension of the solution of the first-order equation $x_{t+1}=a x_{t}$ instead of in Exercise 16 as an afterthought. On the other hand, the solution of the system $d X / d t=$ $A X$ of differential equations in Section 4.7 is immediately given as $X(t)=e^{A t} X(0)$, and the computation for $e^{A t}$ is shown in Appendix 4.14.1. The discrete Putzer algorithm for computing $A^{t}$ can be found in Elaydi's book An Introduction to Difference Equations [5], which is a very good choice to complement the textbook in many aspects. This is one of the cases where the similarities between the solutions discrete and continuous models could be unified by the instructor.

3. The words "density" and "population" are used interchangeably, where either word might mean an unspecified normalization of the actual population. For example, in Example 2.15 , a simple predator-prey model, the variable $x_{t}$ is described as the "prey population" instead of a certain multiple of the population density. Other hidden normalizations plague this example and others. While the convention is perfectly acceptable for technical papers, it is somewhat confusing for the students who are likely to take the word "density" literally, as a quantity between 0 and 1 . See also "dimensionless form" in the next subsection.

4. The central theme, stability, is sometimes lost in the calculations. It might be helpful to refer to the process by name (especially in systems) before starting the stability calculations. In addition, a simple explanation as to why the condition $\left|f^{\prime}(\bar{x})\right|<1$ for the difference equation $x_{t+1}=f\left(x_{t}\right)$ and the condition $f^{\prime}(\bar{x})<0$ for the differential equation $x^{\prime}(t)=f(x)$ (where $\bar{x}$ denotes an equilibrium solution) are similar for local asymptotic stability would be, in my opinion, more to the point than the long explanation before Theorem 2.1, followed by an even longer and complicated epsilon-delta proof; the latter two could have been relegated to the Appendix. Here, once again the instructor has the option of shortening the treatment.

These examples are not exhaustive, but they point to the general problems of the lack of short and simple explanations in favor of long proofs, incomplete treatments of some solution techniques, lack of unity between discrete and continuous models, and sloppy definitions. However, even in this monumental monograph, the experienced or forewarned instructor will have little difficulty navigating the text - it bears repeating - with advance preparation.

\subsubsection{Missed opportunities}

The author seems to be missing some important opportunities to talk about organizing principles and basic assumptions. A few of these are the following.

1. The mass-action principle: The assumption, similar to the principle in chemistry, that random interaction intensity is proportional to the product of the population sizes or densities. The number of random interactions is given by $k x_{t} y_{t}$, where everybody in one population meets everybody else in the other population $k$ times during one time period. 
2. Interaction models as a unified topic: Host-parasite, host-parasitoid, predator-prey, competition, and mutualism models are all similar and the positive and/or negative effects of interaction on multiple species can be treated more clearly; see the undergraduate text Mathematical Models in Biology [3] by Allman and Rhodes.

3. Changes to the reproduction rate: Many population models have the constant reproduction rate modified by a "mortality factor" or "mortality term" times the present population. This should be emphasized uniformly, not just in a couple of examples.

4. Frequency-period relationship: From Section 2.10, An Example: Epidemic model: "The parameter $\gamma$ is the probability of recovery. The ratio $1 / \gamma$ is the average length of the infectious period when there are no deaths..." A little explanation of this recurring theme may be in order. For example, an instructor might add: "Period" is the duration of one cycle in a repeating event, and "frequency" is the number of occurrences of a repeating event per unit time. Clearly they are reciprocals of each other. One individual experiences one recovery in $D$ time units, and the number of recoveries in one time unit is $1 / D=\gamma$.

5. Threshold values: In the same epidemic (SIR) model, the threshold parameter $\mathcal{R}_{0}$ is described as the average number of secondary infections caused by an infected individual. The idea of "lifetime damage or good deeds by one individual" comes up in many disguises but is not isolated as a unifying principle. For example, the parameter $R_{0}$ in Leslie's agestructured model is the average number of female offspring a female has in her lifetime.

6. Dimensionless form of equations or systems: This simplification is first referred to briefly in Section 2.6 on the approximate (discrete) logistic equation, then shows up in more detail, but not with much explanation, in other places, such as Section 6.5 on the (continuous) spruce budworm model.

The instructor should be on the lookout for the above and other organizing principles that may be hidden the first time a topic is introduced but may be explained in full in a later section.

\subsubsection{More Background}

Depending on the topics and examples to be covered, a little bit more background than calculus, differential equations, and linear algebra may be required. Complicated epsilon-delta techniques and concepts such as limit inferior from real analysis are especially ubiquitous. The instructor should also be prepared to insert short introductory or refreshment segments from other disciplines as needed. For example, the treatment of Leslie's age-structured model requires some graph theory terminology. Elaydi's book Discrete Chaos [6] can be used to supplement references to chaos in a compact manner. Dimensionless forms of equations may or may not have been explained adequately in physics or differential equations courses, thus, a short detour may be in order. There is a tiny amount of statistics as well (e.g., the Poisson distribution discussed in the Nicholson-Bailey model of Section 3.3). Towards the end of the text, biochemistry makes an entrance in the well-known chemostat model involving Michaelis-Menten kinetics (Section 6.7.1), and the van der Pol equation takes us into the realm of modeling the action potentials of neurons. I have found it especially difficult to motivate the students in MAT 442 in these two examples (hence, my experiment with population biology).

The final chapter of the textbook, dealing with partial differential equations, brings its own challenges. Even though some solution techniques are described, the extreme complexity of both the modeling process and the chosen solutions are hard to grasp for students who may have a passing acquaintance with PDE's from a one-semester differential equations course. Since it has applications to population biology (such as critical patch size), the topic of reaction-diffusion equations in Section 7.3 may be chosen as the central theme for PDE's and developed carefully with just enough background and details to keep student fatigue to a minimum. Of course, a second semester of differential equations and/or a course in PDE's may be required as a prerequisite, which is impractical in our case. Whatever course of action is chosen, concepts from biochemistry, physics, and analysis will seep into the 
narrative.

\section{The Examples and Exercises}

\subsection{Pros}

Some examples are very detailed and provide an interesting historical perspective into the origin of the problem. My favorite is the Lotka-Volterra model in Section 6.3 on predatorprey models. The inception of the model by Volterra on a request by an Italian biologist who wanted to understand why predator-type fish proliferated during wartime leads to the Volterra principle, which states that a moderate amount of fishing increases the prey population and decreases the predator population. Conversely, if the amount of fishing is decreased (who wants to venture into the Mediterranean with all the U-boats about?), then the predator population increases and the prey population decreases. A modern application of this principle is indiscriminate spraying with pesticides, which kill pests and their (introduced) predators in equal proportions: the prey-pest population will increase, which makes the point of spraying moot. The instructor may want to choose such examples or simply provide any missing background by using the extensive bibliography in each chapter. Otherwise, the examples quickly become a series of dry exercises on writing out the variables involved, constructing the mathematical model, doing some algebra to find equilibrium points (or similar), and checking under which conditions stability holds-sometimes in one long paragraph, without mentioning the steps by name. For example, endless material is available online on epidemics, and current or historical events can be incorporated into the examples. The realism of the model is often discussed with great clarity in [1] and various conclusions and variations of the model are introduced. The instructor should make sure that all examples are equally interesting and have some point beyond the mathematics (which is mostly algebra), following the standard set by the Lotka-Volterra model.

There are many problems at the end of each chapter, which are suitable for assigning as homework, and for doing numerical experiments. The latter can be carried out with a simple instrument such as Microsoft Excel or software such as Maple or MATLAB, depending on the instructor's as well as the students' expertise, which I place in the advantage column.

\subsection{Cons}

Occasionally there aren't enough problems on each subtopic for both homework and exams, which places the onus on the instructor to look for or generate more. Many problems involve too much algebra and may require obscure algebraic tricks that are not standard fare, such as Descartes' rule of signs. In fact, the problem may also end with algebra, not having required the student to explain the results. The numerical data have occasional problems, too. In one problem, the author fails to state that the data would first have to be transformed, which perplexed my students. It is essential for the instructor to solve the problems beforehand and consult the Solutions Manual before assigning them from the textbook. Some additions to the problems, in the form of hints, formulas, and new parts requiring an essay, may be beneficial.

Some of the recurring problems with the solved examples are represented in what follows:

1. Definitions may be blurred inside examples. The meaning of the "birth function" in Example - or Subsection - 3.8.4 (the two-sex model) is not explained well; the phrase may mean the number of breeding couples, family units, or a weighted combination of males and females. Instead we have the following: "The number of offspring produced per mating couple depends on the form of the birth function. The birth function .... is also referred to as the marriage function in human demography. ... [C]ertain functional forms are preferred over others." So, what is the birth function?

2. The notation in examples recurring under different conditions (for example, modeled by difference equations versus differential equations, linear versus nonlinear, one variable 
versus many) is not consistent throughout. The quantities $N_{t}$ and $P_{t}$ of prey and predator populations in the discrete case respectively are replaced by $x(t)$ and $y(t)$ in the continuous case. Moreover, the Neubert-Kolt (discrete) model is said to be "related to" the (continuous) Lotka-Volterra model, and there the matter rests: the differences between such examples (for example, what makes the continuous model preferable to the discrete one) are not emphasized enough. Similarly, Leslie's and continuous age-structured models are related by one sentence only. The instructor may want to touch upon the relationships and perhaps to unify the notation.

3. Certain new techniques are introduced not as a separate unit but as part of an example. The cobwebbing method, for example, is first shown in Subsection (or Example) 3.8.4, not to be heard from again until Chapter 2, some 35 pages later. If most students are not likely to have studied a method earlier, then it would help to isolate that method and even prepare a handout that can be referred to anytime. I have addressed the cobwebbing problem by posting a handout that included how to prepare such graphs by the software currently in use.

\section{The Population Biology Route}

In a one-semester elementary biomathematics modeling course similar to MAT 442 that includes a complete introduction to difference equations, the instructor may want to stick to a unifying theme. Population Biology (with Epidemiology) is an obvious choice, in that the subject matter is very relevant to everyday life and numerous models are applicable to it. Moreover, students appreciate any related visual materials in the form of furry animals or disgusting mold! Here is a suggested road plan, with some personal preferences. Note that systems larger than two-dimensional can be entirely avoided.

\section{Introduction}

Preliminary remarks on deterministic modeling and simple examples such as periodically compounded interest, continuously compounded interest, and the equation of motion for free fall. If there is time, the idea of a small number of rules bringing about very complex changes, e.g., the Game of Life.

1. Linear Difference Equations and Systems

Sections 1.2 and 1.3: Definition and types of difference equations, supplemented by Elaydi [5]. First-order linear difference equations (including the non-homogeneous case) and solutions. Uniqueness and long-term behavior of solutions. Skip cobwebbing.

Sections 1.4 and 1.5: Second- and higher-order linear difference equations with constant coefficients, systems of first-order linear difference equations, and the relationship between the two (when is it possible or desirable to convert one into the other?). Introduce the solution of the system $X_{t+1}=A X_{t}$ as $X_{t}=A^{t} X_{0}$ first and discuss techniques of computing powers of matrices. Then consider solutions via eigenvalues and eigenvectors. Describe longterm behavior. Treat the second-order linear case completely and supplement as needed.

Sections 1.6 and 1.7: Leslie's age-structured model. Present all graph-theoretical and related matrix information and theorems up front. Short proofs or demonstrations for $2 \times 2$ Leslie matrices. Origins and applications of the age-structured population problem. Construction of the model. Long-term behavior of the general solution. Specific examples. The inherent net reproduction number $R_{0}$ and its future relevance (threshold values).

2. First-Order Nonlinear Difference Equations and Systems

Section 2.2: Definitions and examples of (first-order) nonlinear difference equations $x_{t+1}=f\left(x_{t}\right)$ and equilibrium points (solutions) of autonomous ones. Examples of computation of equilibrium points. Skip periodic points and systems for now.

Sections 2.2, 2.3, and 2.4: Introduction to local stability using well-known examples such as the Earth's orbit and bowl-marble. Technical definitions for LA, LS, and LAS 
and the differences between LA and LS by examples. The local stability (LAS) criterion $\left|f^{\prime}(\bar{x})\right|<1$ for first-order equations. The nonhyperbolic case if desired. Alternatively, the cobwebbing method.

Section 2.5: Global stability in first-order nonlinear difference equations. Relationship between local and global stability. Play down heavier technical theorems or demonstrate by cobwebbing.

Sections 2.2, 2.3, and 2.4: Definition, examples, and local stability of periodic points (orbits) by analytical and cobwebbing methods.

Sections 2.6 and 2.7: Tidy account of chaos and related topics such as Liapunov exponents and bifurcation theory for first-order nonlinear difference equations $x_{t+1}=f\left(x_{t}\right)$, supplemented by Elaydi [6]. Demonstrate with (and explain the construction of) the discrete logistic model, explaining the concept of the "dimensionless form" of an equation.

Sections 2.2, 2.8, and 2.9: Definition, examples, and local stability of equilibrium points for first-order nonlinear systems. Jury conditions for three equations.

Section 2.10: Epidemic models. History of epidemics. The concepts of mass-action principle and frequency-period. Construction and simplification of various models. Importance of $\mathcal{R}_{0}$, the basic reproduction number. Relationship with $R_{0}$ in Leslie's age-structured model.

3. Applications of Difference Equations

Section 3.2: Single population models. Explanations of "population", "density", and "density-dependent mortality factor" (which is multiplied by a constant reproduction rate).

Introduction to interaction models (see Allman and Rhodes [3]).

Sections 3.3 and 3.4: The Nicholson-Bailey model and its variations in host-parasitoid systems. Reminder of the Poisson distribution if necessary.

Section 3.6: The Neubert-Kot model of predator-prey relationship. New type of bifurcation that only occurs in systems.

Subsection 3.8.4: Two-Sex models. Inadequacy of female-only models, weighted malefemale dominance from Keyfitz [7], the textbook example, various "birth functions", and the plausibility of the harmonic mean for the birth function (think of average speed of round-trip travel in physics).

Section 3.9: Measles model with vaccination. Discussion of the relationship of the birth rate to eradication of disease in the context of the basic reproduction number. Current epidemics and increasing social resistance to vaccination.

4. Linear Ordinary Differential Equations and Systems (with no major biological examples)

Section 4.3: Malthusian growth equation and its solution by separation of variables for introduction. Review of first-order linear differential equations. Solutions using an integrating factor. Example.

Sections 4.4 and 4.5: Higher-order linear (homogeneous) differential equations with constant coefficients. Solutions. State Routh-Hurwitz Criteria in general and prove for $n=2$. Long-term behavior of solutions (Theorem 4.3). Examples.

Sections 4.6, 4.7, 4.14: First-order homogeneous linear system of differential equations $\frac{d X}{d t}=A X$ with constant coefficients. How to convert higher-order equations into first-order systems. Describe several methods of solving such systems: (1) The exponential solution $X(t)=e^{A t} X(0)$, similar to the single-equation case. The matrix $e^{A t}$ can be computed directly from the definition in simple cases, e.g., when $A$ is diagonal, or by computer algebra. (2) Leonard's method for exponentiating an $n \times n$ matrix by solving an $n$ th-order differential equation, when this is an easier option, is described in the Appendix to Chapter 4. (3) If $A$ is diagonalizable, with $A V_{i}=\lambda_{i} V_{i}$, then the general solution is a linear combination of $e^{\lambda_{i}} V_{i}$. If not, generalized eigenvectors can be used. Complex conjugate eigenvalues are easily handled (demonstrate in $2 \times 2$ case).

Sections 5.2, 5.3, and 4.8: Give the definition of equilibrium solutions for a general (nonlinear) system $\frac{d X}{d t}=F(X)$ in Chapter 5. Similarly, discuss the definitions of stability 
and the condition for local stability for one equation (Theorem 5.3 with short feasibility argument). Then go back to introduce the zero solution as the equilibrium solution of a firstorder linear homogeneous system $\frac{d X}{d t}=A X$, including the scalar case. When $\operatorname{det}(A) \neq 0$, the zero vector is the unique equilibrium solution. The study of its stability determines the stability of equilibria of nonlinear systems via Jacobians evaluated at the equilibrium. Six cases for $2 \times 2$ matrices depending on the trace and the determinant. Not proof but justification of the classification by six examples: solve the system $\frac{d X}{d t}=A X$ and match the shape of the solutions to the type of the stability/unstability of the origin. The six matrices can be taken to be

$$
\left(\begin{array}{ll}
1 & 0 \\
0 & 3
\end{array}\right),\left(\begin{array}{ll}
0 & 1 \\
1 & 0
\end{array}\right),\left(\begin{array}{rr}
-1 & 0 \\
0 & -1
\end{array}\right),\left(\begin{array}{rr}
2 & -1 \\
3 & 2
\end{array}\right),\left(\begin{array}{rr}
0 & 1 \\
-1 & 0
\end{array}\right) \text {, and }\left(\begin{array}{rr}
-1 & 1 \\
-1 & -1
\end{array}\right) .
$$

\section{Nonlinear Ordinary Differential Equations and Systems}

Sections 5.2 and 5.3: Repeat definitions of equilibrium solutions of first-order nonlinear autonomous differential equations and systems. State the condition on local stability in the single-equation case (Theorem 5.3) with simple justification. Compare and contrast with the condition for stability in first-order nonlinear difference equations. Applications to Malthusian growth, logistic growth, and the Allee effect (discuss the manifestations resulting from inbreeding, sex ratio fluctuations - as in the Kakapo parrots, easily found on the Internet - and restricted/infrequent mating contact). Gompertz growth can be skipped. Save periodic solutions for later, when they are more relevant.

Section 5.4: Give an alternative visual approach to stability of equilibria in first-order autonomous equations: phase-line diagrams. Compress to a line from the direction field to show the origin of the idea, then draw directly. Re-evaluate logistic growth and Allee effect using phase-line diagrams. Skip direction fields for non-autonomous equations, as they will not be used.

Section 5.5: Reconsider local stability in first-order $2 \times 2$ systems, using the trace and determinant of the Jacobian $J$ at each equilibrium, similar to the zero equilibrium of the $2 \times 2$ linear system. Note the conditions after Theorem 5.4 (also the non-hyperbolic situation, where $J$ has complex conjugate eigenvalues with zero real part) where the results cannot be compared to the linear case and stability is indeterminate. Include the predator-prey model of Example 5.11.

Give the general condition for local stability in first-order autonomous nonlinear systems of larger size, if desired (Theorem 5.5 in Section 5.5), and skip the example.

Section 5.6: Phase-plane analysis for two-dimensional autonomous systems for a visual determination of stability/unstability types. Again, there are cases where we cannot determine whether the equilibrium solution is a neutral center (with "periodic" solutions) or a spiral of some sort.

Sections 5.2 and 5.7: Define a periodic solution of an autonomous system. Show that the autonomous differential equation $\frac{d x}{d t}=f(x)$, where $f$ is continuous on the real line, cannot have periodic solutions (Theorem 5.2). Also exhibit the periodic solution in Example 5.4. Briefly cover Poincaré-Bendixson Theorem, Poincaré-Bendixson Trichotomy, Bendixson's Criterion, and Dulac's Criterion; reminder of Green's Theorem in the plane. Do Examples 5.14-5.17.

Section 5.8: Consider the Hopf Bifurcation Theorem for a system with two equations, which states sufficient conditions for the existence of periodic solutions. Do Examples 5.195.21 .

6. Biological Applications of Differential Equations

Section 6.2: Harvesting a single population: desire for maximum sustainable yield; logistic growth model, modified via strategies of constant effort or constant yield; comparison of the recovery times for the two models. 
Section 6.3: The Lotka-Volterra predator-prey model. History of Volterra's involvement. Construction, equilibria, and stability for the model. Theorem 6.1: each solution with positive initial conditions is periodic (the proof is lengthy and may be demonstrated by a slide show). Adding effects of constant-rate fishing, we obtain Volterra's principle, with its solution of the original problem (proliferation of predator fish during wartime) and current applications (futility of spraying pest insects in addition to introducing natural predators).

Section 6.3: Discuss the shortcomings of the Lotka-Volterra model. Formulate other predator-prey models, with an emphasis on functional and numerical response.

Subsection 6.4.1: Study the two-species competition model and the founder's effect using prepared slides, which may help ease the tedium. Explore real biological examples of some of the four cases.

Section 6.8: Revisit epidemic models. Consider the effects of vaccination and herd immunity. Compare with discrete epidemic models. The "zombie infection" paper [8] by Munz et al. can be introduced at this point to the delight of all students: again, a slide show (enhanced with some gory pictures) is highly recommended. This may also be saved for the last day of class, following the end of the single PDE model.

7. One Example of Modeling with Partial Differential Equations

First paragraph of Section 7.1: Provide a short introduction for the necessity of modeling with PDE's.

Section 7.3: Give an introduction to reaction-diffusion equations (see additional references by Allen in Section 7.3), where "diffusion" is the population's random diffusion into the spatial domain and "reaction" is the population growth, chosen to be of exponential type (due to the phytoplankton example to be studied). The spatial domain is fixed as $[0, L] \subset \mathbf{R}$, and the boundary condition is Dirichlet. The solution is described in Example 7.6.

Section 7.5: Consider critical patch size. Only the first part of the example, with finite spatial domain $[0, L]$, will be sufficient.

\section{Conclusion}

As with any highly technical course, I found that relevance, humor, rich visuals, and the occasional guest speaker were essential to breaking the monotony. To counteract the fast pace of flow of new information, I encouraged my students to study the contents of each unit before class, solve some preliminary problems, and actively participate in the lecture. In my experience, weekly homework was a must, with my lecture notes indicating at which point the students should attempt a particular problem set.

The reasons why I chose to examine Allen's textbook [1] over Edelstein-Keshet's [4] were mostly personal; the size of the Errata that was displayed (by necessity) in the front matter of the latter book, as well as the truncated treatment of stability definitions, slightly nudged me in the other direction. Otherwise, I found both books to be almost equally suitable for MAT 442, to the extent that I made few changes to my posted lecture notes when switching texts, and often used each book to enrich the notes prepared for the other.

\section{References}

[1] L. J. S. Allen, 2007. An Introduction to Mathematical Biology. Upper Saddle River, NJ: Pearson.

[2] L. J. S. Allen, 2010. An Introduction to Stochastic Processes with Applications to Biology, 2nd edition. Boca Raton: Chapman \& Hall/CRC.

[3] E. S. Allman and J. A. Rhodes, 2003. Mathematical Models in Biology: An Introduction. Cambridge: Cambridge University Press. 
[4] L. Edelstein-Keshet, 2005. Mathematical Models in Biology. Philadelphia: SIAM.

[5] S. N. Elaydi, 1999. An Introduction to Difference Equations, 2nd edition. New York: Springer.

[6] S. N. Elaydi, 2008. Discrete Chaos: With Applications in Science and Engineering, 2nd edition. Boca Raton: Chapman \& Hall/CRC.

[7] N. Keyfitz, 1972. On the mathematics of sex and marriage, in Proceedings of 6th Berkeley Symposium of Mathematical Statistics and Probability, 4:89-108.

[8] P. Munz et al., 2009. When zombies attack!: Mathematical modelling of a zombie infection, in Infection Disease Modelling Research Progress, Edwards et al. (eds), 133-150. 